\title{
Application of contrast-enhanced ultrasound in the pathological grading and prognosis prediction of hepatocellular carcinoma
}

\author{
Zhidong Xuan, Na Wu, Chao Li, Yongrong Liu \\ Ultrasound Three Department, Cangzhou Central Hospital, Cangzhou, China \\ Contributions: (I) Conception and design: Z Xuan; (II) Administrative support: Z Xuan; (III) Provision of study materials or patients: Z Xuan, N Wu; \\ (IV) Collection and assembly of data: C Li, Y Liu; (V) Data analysis and interpretation: Z Xuan; (VI) Manuscript writing: All authors; (VII) Final \\ approval of manuscript: All authors. \\ Correspondence to: Zhidong Xuan. Ultrasound Three Department, Cangzhou Central Hospital, No. 16 Xinhua Road, Cangzhou, China. \\ Email: jieruchaosheng@163.com.
}

Background: To explore the clinical application value of contrast-enhanced ultrasound (CEUS) in the pathological grading and prognosis prediction of hepatocellular carcinoma (HCC).

Methods: A retrospective analysis was performed of 128 patients with primary HCC who underwent CEUS examination in our hospital from January 2017 to June 2020. Patients were divided into three groups: highly-differentiated group, moderately-differentiated group, and poorly-differentiated group. Quantitative analysis of the relationships between the rise time (RT), time to peak (TTP), mean transit time (mTT), intensity maximum (Imax), enhancement rate, and pathological grade of CEUS was performed. In addition, the follow-up patients were divided into a recurrence group and non-recurrence group, and the relationships between RT, TTP, mTT, Imax, and enhancement rate of CEUS were analyzed.

Results: Among the 128 patients, 23 were highly-differentiated, 63 were moderately-differentiated, and 42 were poorly-differentiated. In addition, there were 31 patients in the recurrence group and 97 patients in the non-recurrence group. RT, TTP, and enhancement rate had significant differences in the highlydifferentiated, moderately-differentiated, and poorly-differentiated groups. At the same time, RT and TTP were positively correlated with the differentiation degree, while the enhancement rate was negatively correlated with the differentiation degree. Furthermore, RT, TTP, and enhancement rate were statistically significant for the diagnosis of HCC with high, moderate, and poor differentiation, among which RT had the highest diagnostic accuracy. In the recurrence group, RT, TTP, and Imax were lower than those in the nonrecurrence group, and the enhancement rate was greater than that in the non-recurrence group. Moreover, low levels of RT, TTP, and Imax along with positive microvascular invasion (MVI) and poor differentiation were risk factors for HCC recurrence, and there was no significant relationship between the average tumor diameter and HCC recurrence.

Conclusions: CEUS can significantly show the differences between the RT, TTP, and enhancement rate of HCC across different levels of differentiation. It can also predict whether the disease will relapse. Moreover, low levels of RT, TTP, and Imax as well as positive MVI and poor differentiation can cause the recurrence of HCC.

Keywords: Contrast-enhanced ultrasound (CEUS); hepatocellular carcinoma (HCC); pathological grade; prognosis prediction

Submitted Jun 30, 2021. Accepted for publication Aug 10, 2021.

doi: $10.21037 /$ tcr-21-1264

View this article at: https://dx.doi.org/10.21037/tcr-21-1264 


\section{Introduction}

The incidence of hepatocellular carcinoma (HCC) ranks sixth among malignant tumors and its fatality rate ranks third in terms of cancer-related deaths (1). HCC also ranks second among tumor-related diseases in males (2). There are approximately 750,000 new cases of HCC every year worldwide (3), and the prevalence in men is $2-4$ times that in women (4). Studies have shown that the incidence of HCC is affected by many risk factors $(5,6)$. HCC mostly occurs on the basis of liver cirrhosis, and then undergoes several stages of transformation, from regenerative nodules to dysplasia to liver cancer, which is accompanied by tumor angioarchitecture and blood perfusion $(7,8)$. Early detection and diagnosis of HCC lesions and recurrence are important for improving the long-term prognosis and quality of life of patients. There are many factors affecting the prognosis of HCC, such as the pathological nature and differentiation degree of the tumor. The pathological grade of HCC is an important predictor that reflects the recurrence and survival of HCC patients after surgery or liver transplantation $(9,10)$. Therefore, predicting the pathological grade of liver cancer before surgery can guide the formulation of HCC treatment strategies.

Conventional ultrasound is the primary monitoring method after radical resection of liver cancer, which can diagnose and differentiate tumors in the liver. With the continuous development of ultrasound imaging technology, clinical studies have proven that contrast-enhanced ultrasound (CEUS) can effectively strengthen the twodimensional ultrasound information of the liver (11), reflecting the different blood perfusion of normal tissues and diseased tissues. CEUS is now capable of performing dynamic continuous imaging in real-time through mechanical index and contrast agents to detect tissue microcirculation, providing strong support for the qualitative determination of tumors and providing information of the differentiation status of tumor tissues $(12,13)$. The purpose of this study was to investigate the correlation between the performance of CEUS and the pathological differentiation of primary HCC using CEUS, and to explore the relationships with metastatic prognosis, providing a basis for clinical treatment. We present the following article in accordance with the STARD reporting checklist (available at https://dx.doi.org/10.21037/tcr-211264).

\section{Methods}

\section{General information}

The information of 128 patients with HCC who underwent CEUS examination in our hospital from January 2017 to June 2020 was collected. According to the EdmondsonSteiner tissue differentiation system, patients were divided into a highly-differentiated group, moderately-differentiated group, and poorly-differentiated group. Patients were also divided into a recurrence group and non-recurrence group at follow-up, and all patients were followed up until January 2020. The basic characteristics of patients at admission and follow-up were collected and measured, including age, gender, HBsAg (positive/negative), alpha-fetoprotein (AFP) level $(\leq 20 />20 \mathrm{ng} / \mathrm{mL})$, Child-Pugh grade (A/B/C), tumor number, tumor average diameter, tumor location (left liver lobe/right liver lobe), and microvascular invasion (MVI, positive/negative). All procedures performed in this study involving human participants were in accordance with the Declaration of Helsinki (as revised in 2013). All experiments were approved by the Ethics Committee of Cangzhou Central Hospital (No. 2017-118-01) and informed consent was taken from all the patients.

The inclusion criteria were as follows: (I) patients diagnosed with HCC; (II) maximum tumor diameter $\leq 5 \mathrm{~cm}$; (III) CEUS performed within 1 week before the patient's biopsy or surgery; (IV) all patients and their families were aware of the research content, patients participated voluntarily, and signed an informed consent form.

The exclusion criteria were as follows: (I) patients with neurological diseases and behavioral disorders; (II) patients with other types of liver diseases such as liver cysts; (III) patients with other malignant tumors or distant metastasis of tumors; (IV) patients accepted systemic chemotherapy or local treatment; (V) patients who could not cooperate at follow-up.

\section{Ultrasound examination}

Doppler ultrasound, using a convex probe with a frequency of 1-6 MHz, was performed. First, routine ultrasound scanning was performed, and the size, shape, location, tumor boundary echo, internal echo, and blood flow of the liver tumors were observed and recorded. Subsequently, SonoVue contrast agent was injected from the anterior 
cubital vein, and then the tube was flushed with $5 \mathrm{~mL}$ of saline. Doppler ultrasound was continuously observed for 5 minutes to obtain and evaluate the CEUS image.

Three phases of contrast enhancement were recorded and analyzed: the arterial phase $(10-50 \mathrm{~s}$ after the start of injection), the portal phase (80-120 s after the start of injection), and the anaphase (>120 s), as well as the quantitative analysis of rise time (RT), time to peak (TTP), mean transit time (mTT), intensity maximum (Imax), and enhancement rate.

\section{Statistical analysis}

SPSS 23.0 statistical analysis software was applied to analyze and process the obtained sample data. A $t$-test was used for the comparison of quantitative data, and the $\chi^{2}$ test or Fisher's exact probability method was used for the comparison of qualitative data. $\mathrm{P}<0.05$ indicated that the difference was statistically significant.

\section{Results}

\section{Basic characteristics of patients}

A total of 128 related cases were collected. According to the Edmondson-Steiner tissue differentiation system, 23 cases were highly-differentiated, 63 cases were moderatelydifferentiated, and 42 cases were poorly-differentiated. The comparison of the basic characteristics of patients with different levels of tissue differentiation is shown in Table 1. There were no significant differences in age, gender, HBsAg, AFP level, Child-Pugh grade, number of tumors, and tumor location among the three groups of patients, but there were significant differences in average tumor diameter and MVI.

Of the follow-up patients, there were 31 cases in the recurrence group and 97 cases in the non-recurrence group. The comparison of the basic characteristics of these two groups of patients is shown in Table 2. There were no statistically significant differences in age, gender, HBsAg, AFP level, Child-Pugh grade, tumor number, and tumor location between the two groups, but there were significant differences in average tumor diameter and MVI.

\section{The relationships of CEUS indicators between different levels of HCC differentiation}

The relationships among the quantitative parameters of CEUS in the three groups of highly-differentiated, moderately-differentiated, and poorly-differentiated patients were analyzed. Comparing the three groups, it was found that the differences in mTT and Imax were not statistically significant, but the differences in RT, TTP, and enhancement rate were statistically significant $(\mathrm{P}<0.05)$. The RT and TTP of the highly-differentiated group were higher than those of the moderately-differentiated and poorly-differentiated groups, while the RT and TTP of the moderately-differentiated group were higher than those of the poorly-differentiated group. The enhancement rates of the highly-differentiated and moderately-differentiated groups were lower than the enhancement rate of the poorlydifferentiated group. The specific comparison is shown in Table 3. The CEUS performance and its quantitative analysis curve of HCC patients with different levels of differentiation are shown in Figure 1.

\section{The relationships of CEUS indicators between the recurrence group and non-recurrence group}

There were significant differences in RT, TTP, enhancement rate, and Imax between the recurrence group and the non-recurrence group $(\mathrm{P}<0.05)$. The RT, TTP, and Imax of the recurrence group were lower than those of the non-recurrence group, but the enhancement rate was higher than that of the non-recurrence group (Table 4).

\section{The relationships between $R T$, TTP, enhancement rate, and HCC pathological grade}

The relationships between RT, TTP, enhancement rate, and pathological grade were further analyzed. From Table 5 and Figure 2, it can be seen that RT and TTP were positively correlated with the degree of HCC differentiation. The higher the degree of HCC differentiation, the higher the level of the RT and TTP. The enhancement rate was negatively correlated with the degree of HCC differentiation. The higher the degree of HCC differentiation, the lower the level of the enhancement rate.

\section{The diagnostic value of CEUS indicators in the pathological differentiation of HCC}

Subsequently, we analyzed the diagnostic value of the CEUS quantitative parameters RT, TTP, and enhancement rate in the pathological grading of HCC. The results 
Table 1 Comparison of basic characteristics of patients with different levels of tissue differentiation

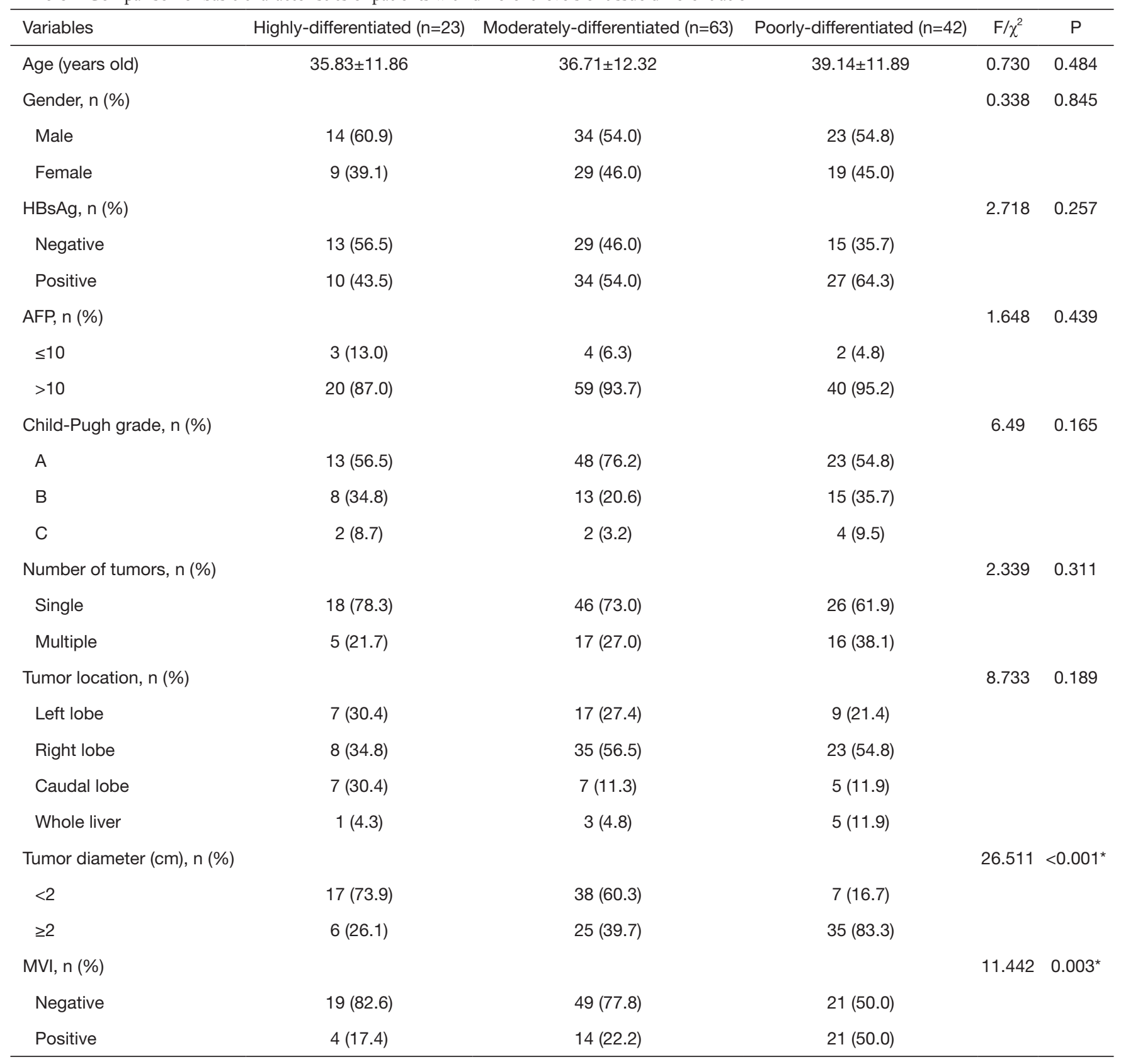

${ }^{*}, \mathrm{P}<0.05$. AFP, alpha-fetoprotein; MVI, microvascular invasion.

in Table 6 showed that RT, TTP, and enhancement rate were statistically significant in the diagnosis of HCC with high differentiation, moderate differentiation, and poor differentiation $(\mathrm{P}<0.05)$. The areas under the curve (AUCs) for the RT, TTP, and enhancement rate were $0.802,0.773$, and 0.775 , respectively, and RT had the greatest diagnostic efficiency.
Multivariate analysis of the relationships between CEUS indicators, tumor diameter, MVI, differentiation degree, and HCC recurrence

A multivariate analysis of the relationships between quantitative parameters of CEUS, average tumor diameter, MVI, and HCC recurrence was performed (Table 7). The results showed that low levels of RT, TTP, and Imax, along with positive 
Table 2 Comparison of basic characteristics between recurrence and non-recurrence patients

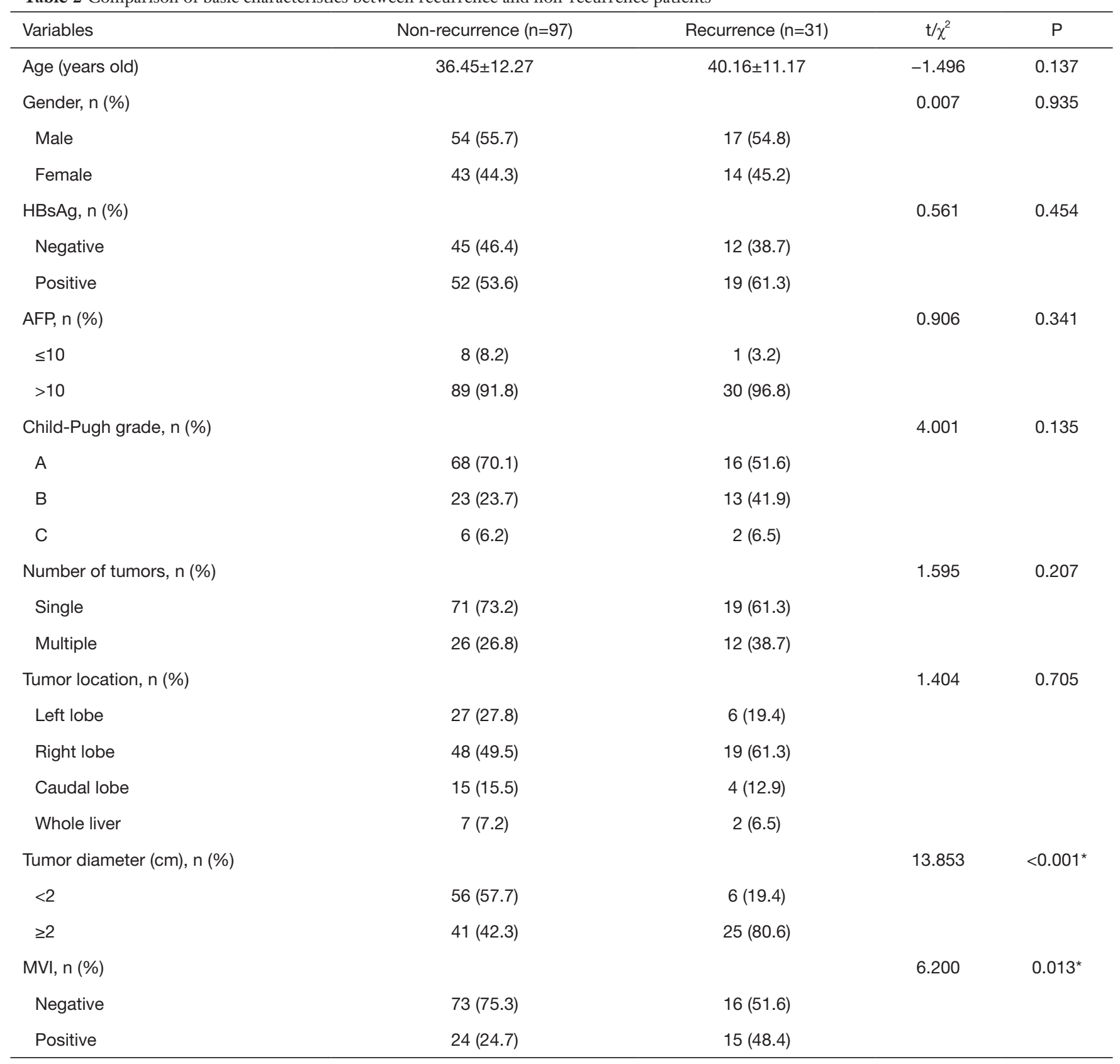

*, $\mathrm{P}<0.05$. AFP, alpha-fetoprotein; MVI, microvascular invasion.

MVI, were risk factors for HCC recurrence, and there was no significant relationship between the differentiation degree, average tumor diameter, and HCC recurrence.

\section{Discussion}

HCC is one of the most common malignant tumors worldwide (1). Among the mortality of tumor-related diseases, the HCC mortality of males ranks second, and the HCC mortality of females ranks sixth (2). The pathological grading of HCC is an important predictor of the recurrence and survival of HCC patients after surgery or liver transplantation $(6,7)$. The risk of recurrence and metastasis of patients with poorly-differentiated HCC is significantly 
Table 3 Comparison of CEUS indicators in patients with different levels of tissue differentiation

\begin{tabular}{|c|c|c|c|c|c|}
\hline Variables & Highly-differentiated $(n=23)$ & Moderately-differentiated $(n=63)$ & Poorly-differentiated $(n=42)$ & $\mathrm{F}$ & $P$ \\
\hline RT & $11.09 \pm 1.87^{\mathrm{b}}$ & $9.86 \pm 2.38^{\mathrm{a}}$ & $6.39 \pm 1.09^{\mathrm{ab}}$ & 56.753 & $<0.001^{*}$ \\
\hline TTP & $12.18 \pm 3.03^{b}$ & $9.19 \pm 2.75^{\mathrm{a}}$ & $7.56 \pm 2.35^{\mathrm{ab}}$ & 22.108 & $<0.001^{*}$ \\
\hline Enhancement rate & $0.63 \pm 0.16$ & $0.76 \pm 0.15$ & $0.80 \pm 0.14^{\mathrm{ab}}$ & 15.000 & $<0.001^{*}$ \\
\hline $\mathrm{mTT}$ & $89.33 \pm 21.76$ & $95.08 \pm 34.01$ & $97.47 \pm 25.26$ & 0.573 & 0.565 \\
\hline
\end{tabular}

Compared with the highly-differentiated group, ${ }^{a} \mathrm{P}<0.05$; compared with the moderately-differentiated group, ${ }^{b} \mathrm{P}<0.05$. ${ }^{*}, \mathrm{P}<0.05$. CEUS, contrast-enhanced ultrasound; RT, rise time; TTP, time to peak; mTT, mean transit time; Imax, intensity maximum.
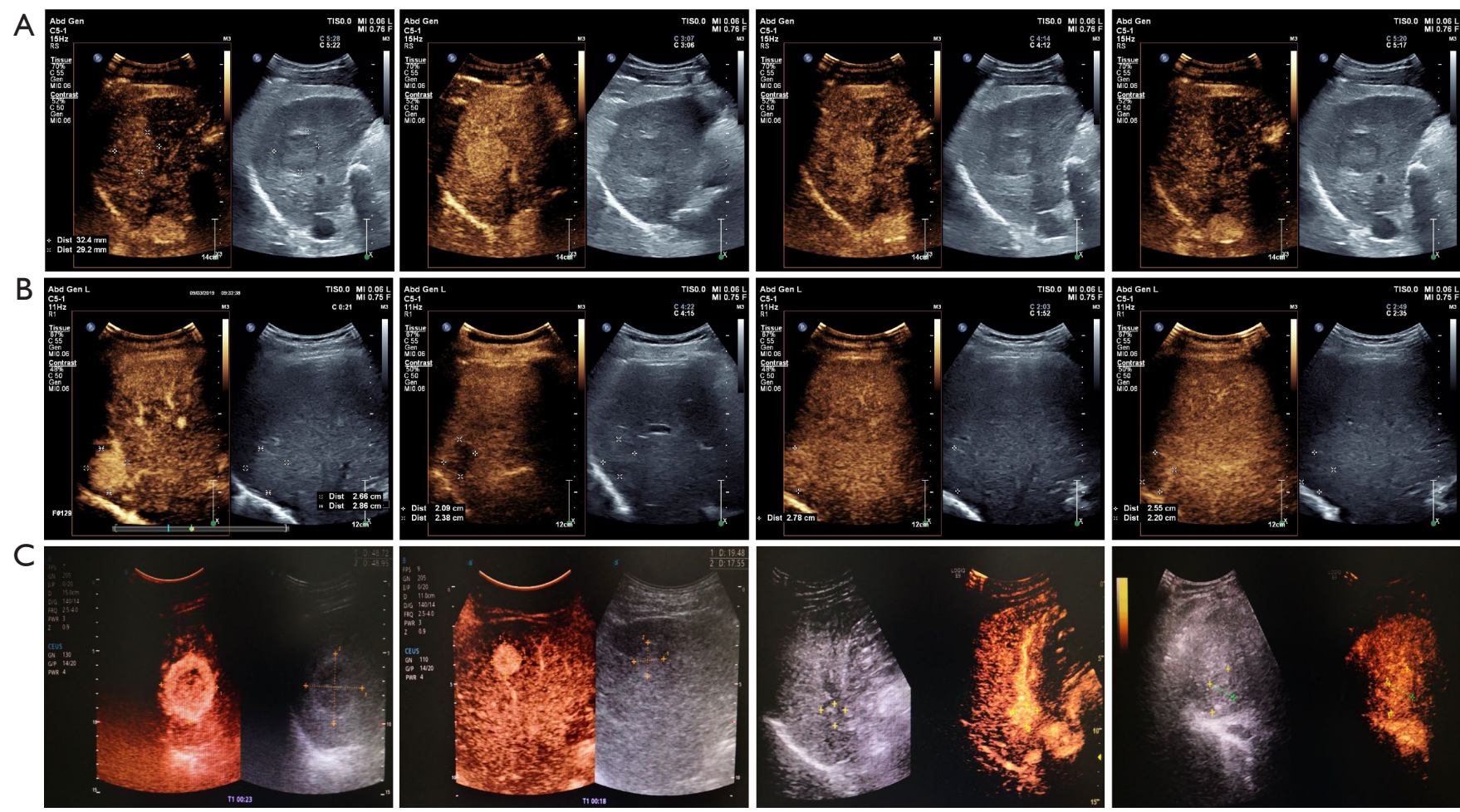

Figure 1 CEUS images and quantitative analysis curves of HCC patients. (A) Highly-differentiated patients; (B) moderately-differentiated patients; (C) poorly-differentiated patients. CEUS, contrast-enhanced ultrasound; HCC, hepatocellular carcinoma.

higher than that of patients with moderately-differentiated HCC $(14,15)$. Accurately assessing the differentiation degree of HCC before treatment can help clinicians formulate and adjust treatment plans, and it is also beneficial for assessing the efficacy and prognosis after treatment. However, the current pathological results of HCC need to be confirmed by needle biopsy or postoperative pathology, which is partly limited.

CT, MRI, and CEUS have the advantages of dynamic and continuous imaging in real-time, which can obtain information during the entire enhancement period (0360 s). However, CT and MRI can only achieve multilayer scanning of the lesion once. CEUS has been recognized by more and more clinicians and patients due to its realtime, low-cost, and low-risk features, and the diagnostic efficacy is comparable to advanced CT and MRI (16-19). Therefore, CEUS can be used to observe the internal blood supply of focal liver lesions. From the perfusion and clearance characteristics of the lesion, the differences in the morphological structure of the diseased tissue cells and the 
Table 4 Comparison of CEUS indicators between the recurrence and non-recurrence group

\begin{tabular}{lcccc}
\hline Variables & Non-recurrence $(\mathrm{n}=97)$ & Recurrence $(\mathrm{n}=31)$ & $\mathrm{t} / \chi^{2}$ & $\mathrm{P}$ \\
\hline RT & $9.66 \pm 2.52$ & $6.70 \pm 1.74$ & 6.064 & $<.001^{*}$ \\
TTP & $9.68 \pm 3.04$ & $7.42 \pm 2.78$ & 3.841 & $<.001^{*}$ \\
Enhancement rate & $0.73 \pm 0.16$ & $0.82 \pm 0.15$ & -2.609 & $0.010^{*}$ \\
mTT & $95.29 \pm 30.54$ & $93.41 \pm 25.62$ & 0.309 & 0.758 \\
Imax & $118.66 \pm 38.66$ & $97.89 \pm 38.77$ & 2.602 & $0.010^{*}$ \\
\hline
\end{tabular}

* $\mathrm{P}<0.05$. CEUS, contrast-enhanced ultrasound; RT, rise time; TTP, time to peak; mTT, mean transit time; Imax, intensity maximum.

Table 5 The relationships between RT, TTP, enhancement rate, and HCC pathological grade

\begin{tabular}{lcc}
\hline \multirow{2}{*}{ Variables } & \multicolumn{2}{c}{ HCC pathological grade } \\
\cline { 2 - 3 } & $\mathrm{r}_{\mathrm{s}}$ & $\mathrm{P}$ \\
\hline RT & 0.6720 & $<0.001^{*}$ \\
TTP & 0.5140 & $<0.001^{\star}$ \\
Enhancement rate & -0.324 & $<0.001^{\star}$ \\
\hline
\end{tabular}

*, $\mathrm{P}<0.05$. RT, rise time; TTP, time to peak; HCC, hepatocellular carcinoma.

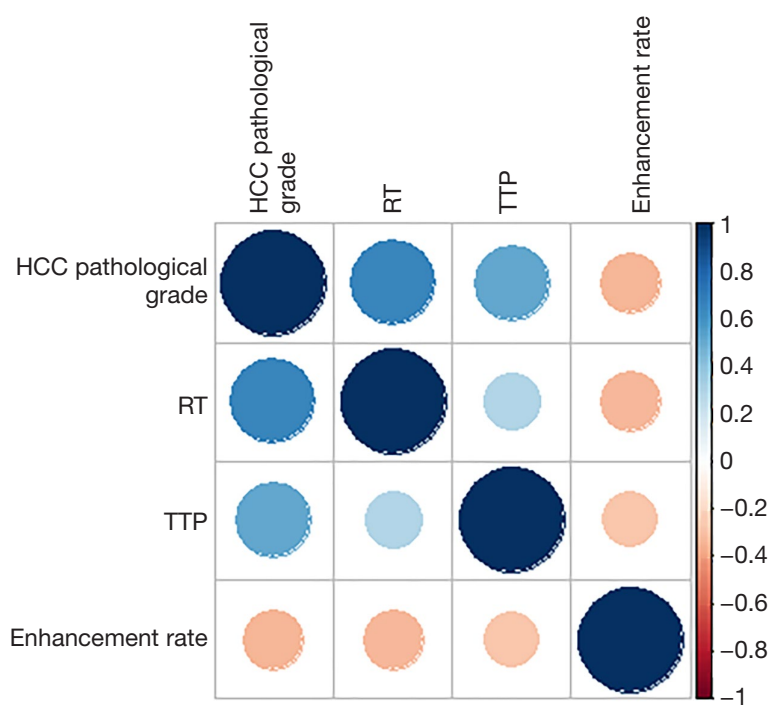

Figure 2 The relationships between RT, TTP, enhancement rate, and HCC pathological grade. RT, rise time; TTP, time to peak; HCC, hepatocellular carcinoma.

internal vascular morphology are obtained, and the nature of the lesion is finally identified, which is more conducive to the comprehensive analysis of the blood perfusion characteristics of the lesion (20-23). Benign and malignant tumors have unique performance on CEUS, which plays an increasingly important role in the identification of benign and malignant liver tumors. There are many different manifestations when using CEUS to examine HCC, the most common of which are "fast in and fast out" and "fast in and slow out/no out". Depending on the degree of contrast intensity, there is a difference of significant enhancement and insignificant enhancement. Nicolau et al. (24) studied the CEUS performance of 104 cases of HCC, and 4 cases with significant enhancement in the arterial phase were all highly-differentiated HCC.

Among the patients in this study, 23 were in the highly-differentiated group, 63 were in the moderatelydifferentiated group, and 42 were in the poorlydifferentiated group. Comparing the three groups, the differences in RT, TTP, and enhancement rates were statistically significant, while the differences in $\mathrm{mTT}$ and Imax were not statistically significant. Among them, the RT and TTP of the highly-differentiated and moderatelydifferentiated groups were higher than those of the poorlydifferentiated group. There were no significant differences in mTT and Imax between the highly-, moderately-, and poorly-differentiated groups. Furthermore, we determined that RT and TTP were positively correlated with the HCC differentiation degree, and the enhancement rate was negatively correlated with the HCC differentiation degree. In addition, the receiver operating characteristic (ROC) curve showed that RT, TTP, and enhancement rate were statistically significant for the diagnosis of HCC of high differentiation, moderate differentiation, and poor differentiation, among which RT had the highest diagnostic accuracy.

Liver cancer is prone to metastasis. Studies have shown that poor prognosis may be related to the size and number of tumors, the patient's own liver function, 
Table 6 The diagnostic value of CEUS indicators in the pathological grading of HCC

\begin{tabular}{lccccc}
\hline Variables & AUC & P & Sensitivity & Specificity & Accuracy \\
\hline RT & 0.802 & $<0.001^{*}$ & 100 & 50.48 & 0.5048 \\
TTP & 0.773 & $<0.001^{*}$ & 78.26 & 86.67 & 0.6493 \\
Enhancement rate & 0.775 & $<0.001^{*}$ & 73.91 & 88.57 & 0.6248 \\
\hline
\end{tabular}

*, $\mathrm{P}<0.05$. CEUS, contrast-enhanced ultrasound; HCC, hepatocellular carcinoma; AUC, area under the curve; RT, rise time; TTP, time to peak.

Table 7 Multivariate analysis of the relationships between CEUS indicators, tumor diameter, MVI, differentiation degree, and HCC recurrence

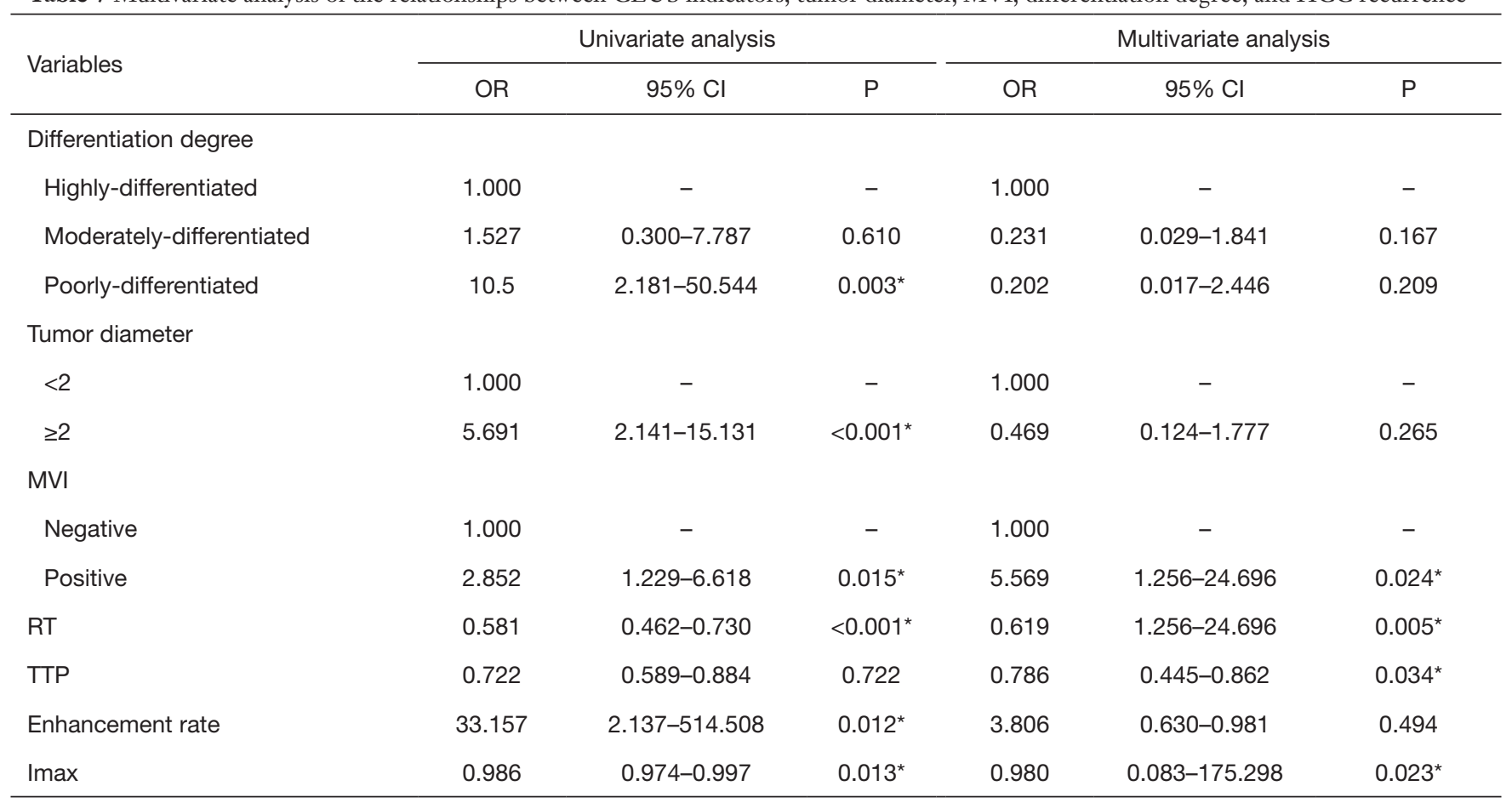

*, $\mathrm{P}<0.05$. CEUS, contrast-enhanced ultrasound; HCC, hepatocellular carcinoma; MVI, microvascular invasion; RT, rise time; TTP, time to peak; Imax, intensity maximum.

and the differentiation degree of the tumor, but there are not many studies investigating the association with imaging characteristics (25-27), making the prognosis prediction of HCC a critical step. In this study, we found that the RT, TTP, and Imax of the recurrence group were significantly lower than the non-recurrence group, while the enhancement rate was significantly higher than the non-recurrence group. Further multivariate analysis showed that low levels of RT, TTP, and Imax along with positive MVI and poor differentiation were risk factors for HCC recurrence, and there was no significant relationship between the average tumor diameter and HCC recurrence.

\section{Conclusions}

In summary, CEUS can significantly show the differences between the RT, TTP, and enhancement rate of HCC across different levels of differentiation, and it can also predict the recurrence of HCC. Low-level RT, TTP, and Imax as well as positive MVI and poor differentiation can cause the recurrence of HCC.

\section{Acknowledgments}

Funding: None. 


\section{Footnote}

Reporting Checklist: The authors have completed the STARD reporting checklist. Available at https://dx.doi. org/10.21037/tcr-21-1264

Data Sharing Statement: Available at https://dx.doi. org/10.21037/tcr-21-1264

Conflicts of Interest: All authors have completed the ICMJE uniform disclosure form (available at https://dx.doi. org/10.21037/tcr-21-1264). The authors have no conflicts of interest to declare.

Ethical Statement: The authors are accountable for all aspects of the work in ensuring that questions related to the accuracy or integrity of any part of the work are appropriately investigated and resolved. All procedures performed in this study involving human participants were in accordance with the Declaration of Helsinki (as revised in 2013). The study was approved by the Ethics Committee of Cangzhou Central Hospital (No. 2017-118-01) and informed consent was taken from all the patients.

Open Access Statement: This is an Open Access article distributed in accordance with the Creative Commons Attribution-NonCommercial-NoDerivs 4.0 International License (CC BY-NC-ND 4.0), which permits the noncommercial replication and distribution of the article with the strict proviso that no changes or edits are made and the original work is properly cited (including links to both the formal publication through the relevant DOI and the license). See: https://creativecommons.org/licenses/by-nc-nd/4.0/.

\section{References}

1. Bray F, Ferlay J, Soerjomataram I, et al. Global cancer statistics 2018: GLOBOCAN estimates of incidence and mortality worldwide for 36 cancers in 185 countries. CA Cancer J Clin 2018;68:394-424.

2. Torre LA, Bray F, Siegel RL, et al. Global cancer statistics, 2012. CA Cancer J Clin 2015;65:87-108.

3. Maluccio M, Covey A. Recent progress in understanding, diagnosing, and treating hepatocellular carcinoma. CA Cancer J Clin 2012;62:394-9.

4. El-Serag HB. Hepatocellular carcinoma. N Engl J Med 2011;365:1118-27.

5. Wang F, Wang G, Liu T, et al. B7-H3 was highly expressed in human primary hepatocellular carcinoma and promoted tumor progression. Cancer Invest 2014;32:262-71.

6. Gitto S, Di Sandro S, Magistri P, et al. Hepatocellular carcinoma and liver transplant: beyond the Milan criteria and the risk of "short-blanket" syndrome. Hepatobiliary Surg Nutr. 2020;9:518-21.

7. Bhat A, Layfield LJ, Tewari SO, et al. Solitary fibrous tumor of the ischioanal fossa-a multidisciplinary approach to management with radiologic-pathologic correlation. Radiol Case Rep 2018;13:468-74.

8. Fong ZV, Tanabe KK. The clinical management of hepatocellular carcinoma in the United States, Europe, and Asia: a comprehensive and evidence-based comparison and review. Cancer 2014;120:2824-38.

9. Shibata J, Kawai K, Nishikawa T, et al. Prognostic impact of histologic type in curatively resected stage IV colorectal cancer: a Japanese multicenter retrospective study. Ann Surg Oncol 2015;22 Suppl 3:S621-9.

10. Yokota K, Kataoka Y. Fabrication of three-dimensional $\mathrm{Al} 2 \mathrm{O} 3-\mathrm{ZrO} 2-\mathrm{TiO} 2$ ceramics by digital light processing type vat photopolymerization. Journal of the Society of Powder Technology Japan 2019;56:261-6.

11. Lam S, Zuo T, Ho M, et al. Review article: fungal alterations in inflammatory bowel diseases. Aliment Pharmacol Ther 2019;50:1159-71.

12. Chen Y, Tang L, Du Z, et al. Factors influencing the performance of a diagnostic model including contrastenhanced ultrasound in 1023 breast lesions: comparison with histopathology. Ann Transl Med 2019;7:647.

13. Mudambi K, Sandberg J, Bass D, et al. Contrast enhanced ultrasound: comparing a novel modality to MRI to assess for bowel disease in pediatric Crohn's patients. Transl Gastroenterol Hepatol 2020;5:13.

14. Pan X, Huang L, Mo D, et al. SNP rs2240688 in CD133 gene on susceptibility and clinicopathological features of hepatocellular carcinoma. Transl Cancer Res 2020;9:5940-48.

15. Mao K, Yan Y, Zhang J, et al. The impact of liver resection on survival outcomes of hepatocellular carcinoma patients with extrahepatic metastases: a propensity score matching study. Cancer Med 2018;7:4475-84.

16. Zhao C, Yu D, Kang K, et al. Role of contrast-enhanced ultrasound sonography in the medical diagnostics of the disease activity in patients with Takayasu arteritis. IEEE Access 2019;7:23240-8.

17. Wong GYM, Kumar R, Beeke C, et al. Survival outcomes for patients with indeterminate 18 FDG-PET scan for 
extrahepatic disease before liver resection for metastatic colorectal cancer: a retrospective cohort study using a prospectively maintained database to analyze survival outcomes for patients with indeterminate extrahepatic disease on 18FDG-PET scan before liver resection for metastatic colorectal cancer. Ann Surg 2018;267:929-35.

18. Kierans AS, Kang SK, Rosenkrantz AB. The diagnostic performance of dynamic contrast-enhanced MR imaging for detection of small hepatocellular carcinoma measuring up to $2 \mathrm{~cm}$ : a meta-analysis. Radiology 2016;278:82-94.

19. Zhang CY, Fu Y, Li XD, et al. Advances in imaging diagnosis of liver cancer. Journal of Clinical Hepatology 2017;33:1266-9.

20. Arita J, Ono Y, Takahashi M, et al. Usefulness of contrastenhanced intraoperative ultrasound in identifying disappearing liver metastases from colorectal carcinoma after chemotherapy. Ann Surg Oncol 2014;21 Suppl 3:S390-7.

21. Hiroyoshi J, Yamashita S, Tanaka M, et al. Contrastenhanced intraoperative ultrasound in the resection of colorectal liver metastases with intrabiliary growth. Clin J Gastroenterol 2018;11:348-53.

22. Rappeport ED, Loft A, Berthelsen AK, et al. Contrastenhanced FDG-PET/CT vs. SPIO-enhanced MRI vs. FDG-PET vs. CT in patients with liver metastases from

Cite this article as: Xuan Z, Wu N, Li C, Liu Y. Application of contrast-enhanced ultrasound in the pathological grading and prognosis prediction of hepatocellular carcinoma. Transl Cancer Res 2021;10(9):4106-4115. doi: 10.21037/tcr-21-1264 colorectal cancer: a prospective study with intraoperative confirmation. Acta Radiol 2007;48:369-78.

23. Shonaka T, Inagaki M, Shomura H, et al. Contrast enhanced intraoperative ultrasound is effective for liver metastasis of colorectal cancer. The Japanese Journal of Gastroenterological Surgery 2015;48:1-7.

24. Nicolau C, Catalá V, Vilana R, et al. Evaluation of hepatocellular carcinoma using SonoVue, a second generation ultrasound contrast agent: correlation with cellular differentiation. Eur Radiol 2004;14:1092-9.

25. Vikram R, Ng CS, Tamboli P, et al. Papillary renal cell carcinoma: radiologic-pathologic correlation and spectrum of disease. Radiographics 2009;29:741-54; discussion 755-7.

26. Cheng SH, Guo XP. Effect of ultrasound-guided transversus abdominis plane block analgesia after caesarean delivery. Laboratory Medicine and Clinic 2017;14:3214-5, 3218.

27. Li B, Hu WN. Efficacy and Safety Evaluation of threedimensional stereotactic CT guided 125I seed implantation in the treatment of primary liver cancer. Labeled Immunoassays and Clinical Medicine 2016;23:1294-8.

(English Language Editor: C. Betlazar-Maseh) 